\title{
Towards New Technical Indicators for Trading Systems and Risk Management
}

\author{
Michel FLIESS * Cédric JOIN ** \\ * INRIA-ALIEN \& LIX (CNRS, UMR 7161) \\ École polytechnique, 91128 Palaiseau, France \\ Michel.Fliess@polytechnique.edu \\ ** INRIA-ALIEN \&f CRAN (CNRS, UMR 7039) \\ Nancy-Université, BP 239, 54506 Vandœuvre-lès-Nancy, France \\ Cedric.Join@cran.uhp-nancy.fr
}

\begin{abstract}
We derive two new technical indicators for trading systems and risk management. They stem from trends in time series, the existence of which has been recently mathematically demonstrated by the same authors (A mathematical proof of the existence of trends in financial time series, Proc. Int. Conf. Systems Theory: Modelling, Analysis and Control, Fes, 2009), and from higher order quantities which replace the familiar statistical tools. Recent fast estimation techniques of algebraic flavor are utilized. The first indicator tells us if the future price will be above or below the forecasted trendline. The second one predicts abrupt changes. Several promising numerical experiments are detailed and commented.
\end{abstract}

\section{INTRODUCTION}

\subsection{Generalities}

This communication provides two new efficient technical indicators for trading systems and risk management, by taking advantage of the trends in financial time series. Remember that a precise mathematical definition of trends as well as a proof of their existence have only been recently given (Fliess \& Join [2009a]). It confirms thus the most basic assumption of technical analysis, which plays a key rôle among many traders and financial professionals (see, e.g., (Aronson [2007], Béchu, Bertrand \& Nebenzahl [2008], Bollinger [2002], Kaufman [2005], Kirkpatrick \& Dahlquist [2006], Lo \& Hasanhodzic [2009], Murphy [1999]) and the references therein).

\subsection{Brief description of our new indicators and of their properties}

The definition and the existence of trends in (Fliess \& Join [2009a]) is deduced from the Cartier-Perrin additive decomposition (Cartier \& Perrin [1995]) of a given time series $f(t)$, which reads under a very weak integrability condition:

$$
f(t)=f_{\text {trend }}(t)+f_{\text {fluctuation }}(t)
$$

$f_{\text {trend }}(t)$ and $f_{\text {fluctuation }}(t)$ are respectively the trend and a "quickly fluctuating" function around 0 . Introduce now the time series

$$
\operatorname{ABS}-D I F F_{\iota}(t)=\left|\hat{f}_{\text {trend }}(t+\iota)-f(t)\right|
$$

where

- $\iota \geq 0$,

- $\hat{f}_{\text {trend }}(t+\iota)$ is the forecasted value of $f_{\text {trend }}(t)$ at time $t+\iota$, which is obtained as in (Fliess \& Join [2009a]).

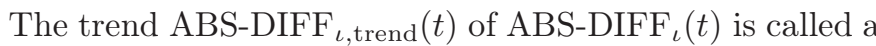
higher-order trend associated to the time series $f(t)$. Con- struct now the quantities, which are vaguely reminiscent of the classic statistical skewness,

$$
\operatorname{SKEW}_{\iota}(t)=\left(\frac{\hat{f}_{\text {trend }}(t+\iota)-f(t)}{\widehat{\operatorname{ABS-DIFF}}_{\iota, \text { trend }}(t+\iota)}\right)^{3}
$$

where $\widehat{\mathrm{ABS}^{-D I F F}} \mathrm{~F}_{\iota}$ trend $(t+\iota)$ is the forecasted value of $\operatorname{ABS} \mathrm{DIFF}_{\iota, \text { trend }}(t)$ at time $t+\iota$. Our two indicators are derived from Equations (2) and (3):

(1) The first one yields buy and sell signals by telling if future prices will be above or below the forecasted trendline. It greatly improves previous calculations in (Fliess \& Join [2008b, 2009a]).

(2) The second one is related to risk management, which - is a central theme in modern quantitative finance,

- seems to be more or less ignored until now in the existing research literature related to technical analysis (see, however, Dacorogna, Gençay, Müller, Olsen \& Pictet [2001]).

The kind of volatility which is associated to Equation (2) is exploited, via new algebraic techniques stemming from (Fliess, Join \& Mboup [2010]), in order to forecast abrupt changes in the FOREX. Our promising computer simulations indicate a possible alternative

- not only to

- Monte Carlo techniques (see, e.g., (Glasserman [2004]) and the references therein),

- the well known Value at Risk, or VaR, and its multiple variants (see, e.g., (Jorion [2001]) and the references therein),

- but also to more mathematically oriented works such as (Bouchaud \& Potters [1997-2000], Malevergne \& Sornette [2006], Mandelbrot \& Hudson [2004]).

All those attempts rely on the search for a "good" probabilistic modeling, which often goes beyond the 
Gaussian paradigm, whereas the need of any probabilistic model is bypassed here (see also Section 5.2 and (Fliess \& Join [2009a])).

\subsection{Organization of the paper}

Section 2 aims at presenting in plain words the CartierPerrin theorem and its utilization. Numerical experiments are provided in Sections 3 and 4 respectively on the position with respect to the forecasted trendline and on the prediction of abrupt changes. Some concluding remarks are discussed in Section 5.

\section{TRENDS AND QUICK FLUCTUATIONS}

We refer to (Fliess \& Join [2009a]) and to (Lobry \& Sari [2008]) for details on the Cartier-Perrin theorem (Cartier \& Perrin [1995]), which is expressed in the framework of nonstandard analysis. We will be employing here a very imprecise language, which hopefully will give to nonexperts an intuitive feeling of what it is all about.

The decomposition in Equation (1) holds if $f(t)$ is integrable. Then,

- the trend $f_{\text {trend }}(t)$ is integrable and almost everywhere continuous;

- the quickly fluctuating function $f_{\text {fluctuation }}(t)$ is defined by the fact that its integral $\int_{a}^{b} f_{\text {fluctuation }}(\tau) d \tau$ over any finite interval $[a, b]$ is infinitesimal, i.e., "very small".

Those quick fluctuations are similar to corrupting additive noises in engineering, and especially in signal processing and in automatic control. The practical calculation of the trend in (Fliess \& Join [2009a]) is therefore achieved via estimation techniques which were developed elsewhere and for other purposes (see (Fliess, Join \& Sira-Ramírez [2008], Mboup, Join \& Fliess [2009]) and the references therein). It should be emphasized that those techniques may be viewed as an extension of the familiar moving average methods which are central in technical analysis (see, e.g., Béchu, Bertrand \& Nebenzahl [2008]).

\section{ABOVE OR BELOW THE FORECASTED TRENDLINE?}

Figure 1 displays the daily stock prices of Arcelor-Mittal from 7 July 1997 until 27 October 2008. ${ }^{1}$ We first estimate and forecast the trend of the daily prices as in (Fliess \& Join [2009a]), i.e., by applying the techniques of (Fliess, Join \& Sira-Ramírez [2008], Mboup, Join \& Fliess [2009]). It yields $\operatorname{ABS}_{-D_{I F F}}(t), \operatorname{ABS}-D I F F_{\iota, \text { trend }}(t)$, $\mathrm{ABS-DIFF}_{\iota \text { trend }}(t+\iota)$, and $\operatorname{SKEW}_{\iota}(t)$, which were defined in Section 1.2. The last quantity tells us if the future price will be above or below the forecasted trendline, if we assume that its sign is the same as the sign of $\hat{f}_{\text {trend }}(t+\iota)-$ $f(t+\iota)$. The meaning of the symbols $\triangle$ and $\nabla$ is obvious. The forecast index $\iota$ is respectively 1,5 , and 10 .

The fact that the results do not deteriorate when the prediction time interval increases might seem rather surprising at first sight. This is explained by a parallel increase

\footnotetext{
1 Those data are borrowed from http://finance. yahoo.com/.
}

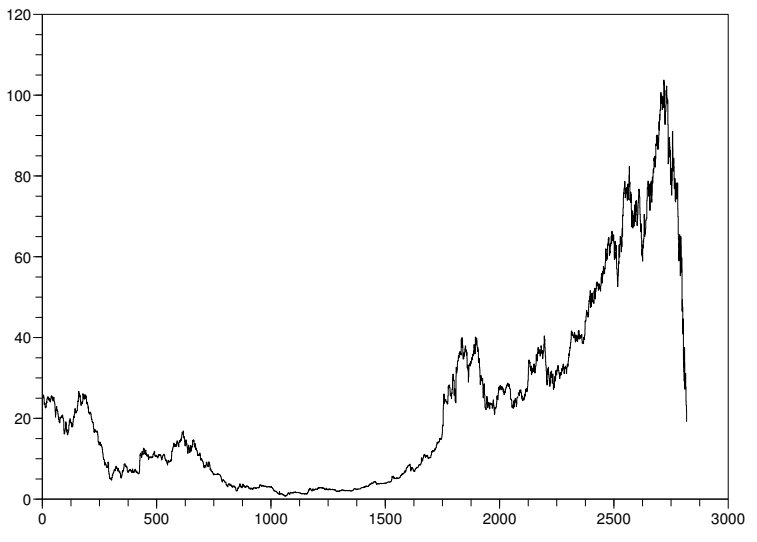

Figure 1. Arcelor-Mittal daily stock prices

of the time interval for determining the trend, i.e., we are not working with the same trends. Our forecasts, which give $75 \%$ of exact results, are quite remarkable.

\section{TOWARDS THE FORECASTING OF ABRUPT CHANGES}

The black lines in Figures 14-(a) represents the exchange rates between US Dollars (USD) and Euros (EUR) during 2000 days, until 27 October 2008. ${ }^{2}$ Our attempt to forecast abrupt changes is based on the following "empirical" fact:

An abrupt change in the FOREX is preceded by the detection of an abrupt change of the "volatility" around the trend.

This general principle is implemented as follows:

We take here as a measure of volatility the quantity

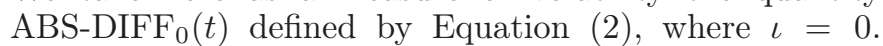
The corresponding signal is very "noisy", and thus difficult to analyze. We therefore replace it by its integral $\int \mathrm{ABS} \mathrm{DIFF}_{0}(\tau) d \tau$, where, according to (Fliess [2006]), the noise is attenuated. Our task boils down to the detection of abrupt changes in the derivative of $\int \mathrm{ABS}-\mathrm{DIFF}_{0}(\tau) d \tau$, which is achieved via

- extrapolation techniques which were already utilized in Section 3 and in (Fliess \& Join [2009a]),

- the methods developed in (Fliess, Join \& Mboup [2010]).

Figure 14 depicts the evolution and the forecasting of the various quantities considered above. Let us clarify our results thanks to Figures 14-(g) and 14-(h):

- In Figure 14-(g) one change-point is detected 10 days ahead but another one is missed.

- When the detection threshold becomes lower, Figure 14-(h) shows that both change-points are detected again 10 days ahead.

2 Those data are borrowed from the European Central Bank. 


\section{CONCLUSION}

\subsection{New indicators}

The efficiency of our two indicators should of course be confirmed by further numerical and experimental studies, where high frequency data ought to be considered for the FOREX. Our second indicator will be completed in a near future in order to predict if the abrupt change will be a sudden increase or reduction. Many more indicators might obviously be derived along the same lines, i.e., by taking advantage of well chosen higher order trends.

\subsection{Probability and statistics}

We already criticized in (Fliess \& Join [2009a]) the use of probability theory in quantitative finance. It is indeed difficult, if not impossible, to model the inherent uncertainty of such complex social behaviors via precise probability laws. ${ }^{3}$ We go here one step further and replace the traditional statistical tools by new quantities like those defined by Equations (2) and (3). It should open new ways for investigating time series in general, in a model-free setting (see already (Fliess \& Join [2008b]) for a first draft, and (Fliess \& Join [2008a, 2009b]) for model-free control).

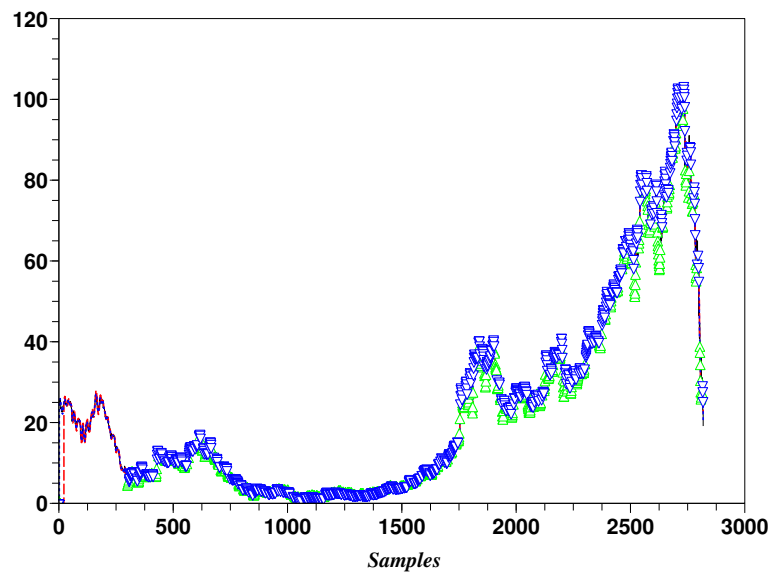

Figure 2. Prices (black,-), filtered signal (blue,.), 1 day forecast (red, - -), price's forecast higher than the predicted trend (green $\triangle$ ), price's forecast lower than the predicted trend (blue $\nabla)(1$ day)

\section{REFERENCES}

D. Aronson. Evidence-Based Technical Analysis. Wiley, 2007.

T. Béchu, E. Bertrand, J. Nebenzahl. L'analyse technique (6 $6^{e}$ éd.). Economica, 2008.

J. Bollinger. Bollinger on Bollinger Bands. McGraw-Hill, 2002.

\footnotetext{
3 The epistemological difficulties related to probability in pure and applied sciences are discussed by Jaynes [2003] (the authors thank Prof. A. Richard for pointing out to them this reference). See also Daloz [1973] (the authors thank J.-E. Laisney for pointing out to them this reference) and Mouchot [1996] for interesting discussions on the rôle of probability in finance and econometrics.
}

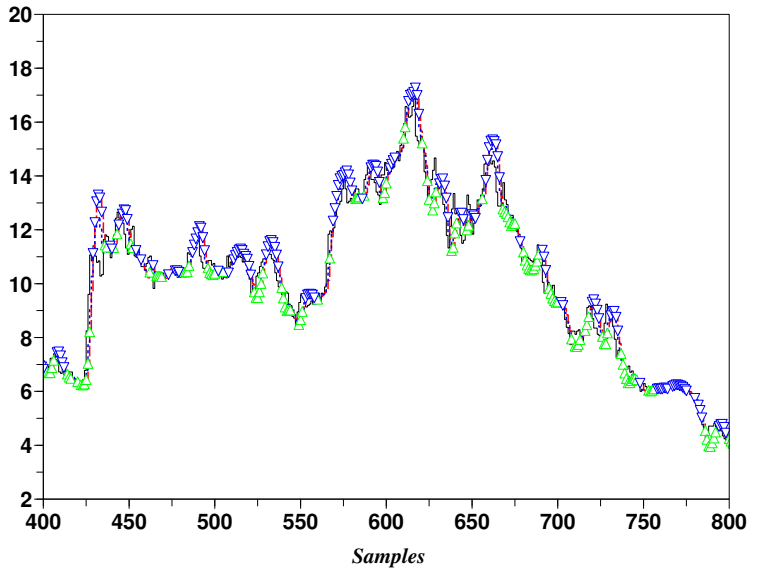

Figure 3. Zoom of Figure 2 (1 day)

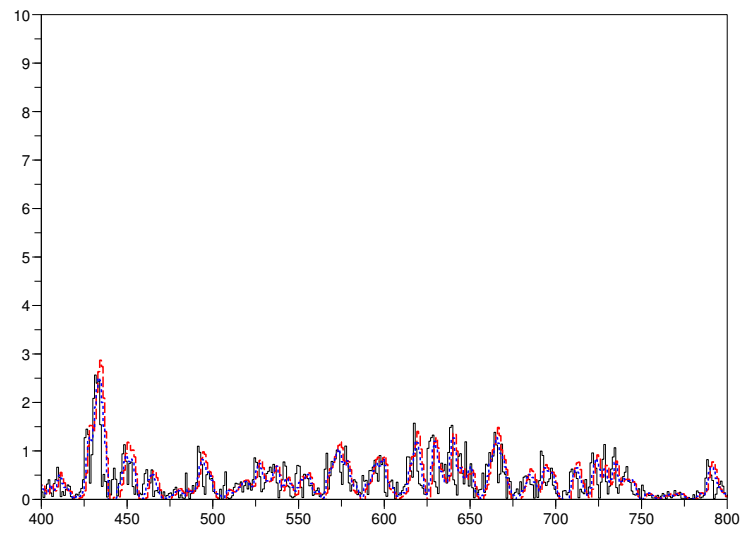

Figure 4. ABS-DIFF 1 (black,-), filtered signal (blue,.), 1 day forecast (red, - -) (1 day)

J.-P. Bouchaud, M. Potters. Théorie des risques financiers. Eyrolles, 1997. English translation: Theory of Financial Risks. Cambridge University Press, 2000.

P. Cartier, Y. Perrin. Integration over finite sets. In F. \& M. Diener, editors. Nonstandard Analysis in Practice, pages 195-204. Springer, 1995.

M.M. Dacorogna, R. Gençay, U. Müller, R.B. Olsen, O.V. Pictet. An Introduction to High Frequency Finance. Academic Press, 2001.

J.-P. Daloz. Le hasard et les cours boursiers. Cujas, 1973. M. Fliess. Analyse non standard du bruit. C.R. Acad. Sci. Paris Ser. I, 342:797-802, 2006.

M. Fliess, C. Join. Commande sans modèle et commande à modèle restreint. e-STA, $5\left(\mathrm{n}^{\circ} 4\right): 1-23,2008 \mathrm{a}$ (available at http: //hal.inria.fr/inria-00288107/en/).

M. Fliess, C. Join. Time series technical analysis via new fast estimation methods: a preliminary study in mathematical finance. Proc. $23^{\text {rd }}$ IAR Workshop Advanced Control Diagnosis (IAR-ACD08), Coventry, 2008b (available at http://hal.inria.fr/inria-00338099/en/). 


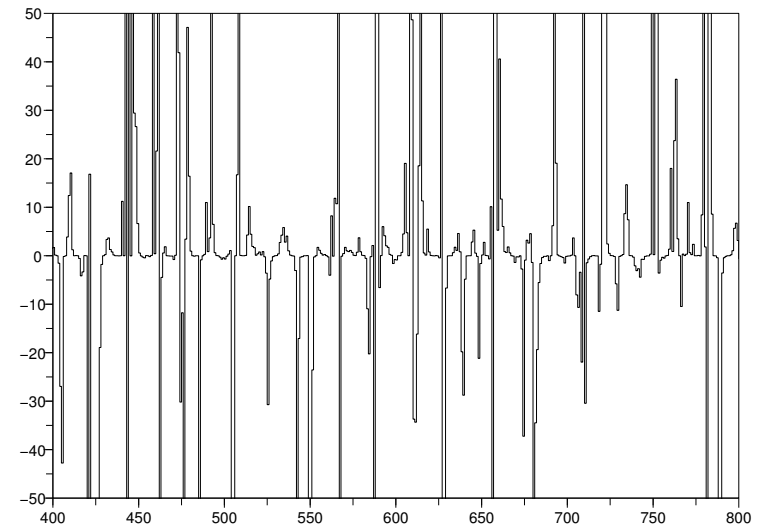

Figure 5. SKEW 1 (1 day)

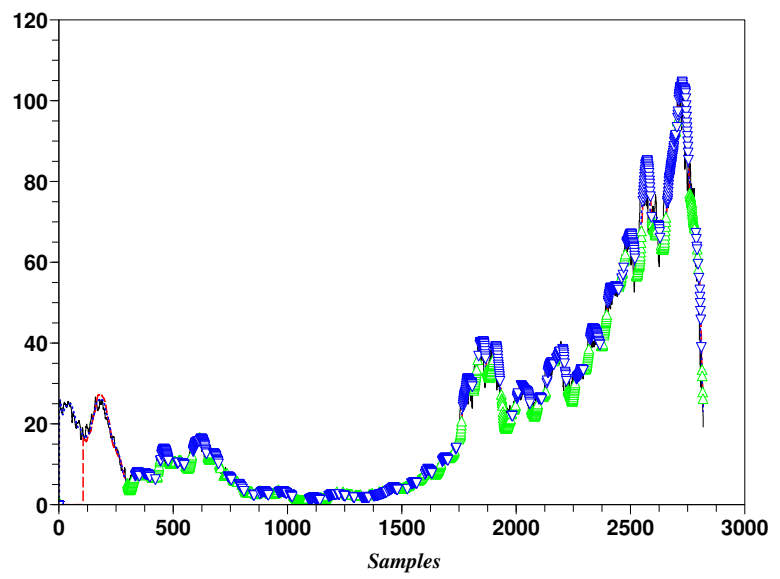

Figure 6. Prices (black,-), filtered signal (blue,.), 5 day forecast (red, - -), forecast of the prices above the predicted trend (green $\triangle$ ), forecast of the prices below the predicted trend (blue $\nabla)$ ( 5 days)

M. Fliess, C. Join. A mathematical proof of the existence of trends in financial time series. Proc. Int. Conf. Systems Theory: Modelling, Analysis and Control, Fes, 2009a (available at http://hal.inria.fr/inria-00352834/en/).

M. Fliess, C. Join. Model-free control and intelligent PID controllers: towards a possible trivialization of nonlinear control?. Proc. $15^{\text {th }}$ IFAC Symp. System Identif. (SYSID 2009), Saint-Malo, 2009b (available at http://hal.inria.fr/inria-00372325/en/).

M. Fliess, C. Join, M. Mboup. Algebraic change-point detection. Applicable Algebra Engin. Communic. Comput., accepted for publication, 2010 (soon available at http://hal.inria.fr/).

M. Fliess, C. Join, H. Sira-Ramírez. Nonlinear estimation is easy. Int. J. Model. Identif. Control, 4:12-27, 2008 (available at http://hal.inria.fr/inria-00158855/en/).

P. Glasserman. Monte Carlo Methods in Financial Engineering. Springer, 2004.

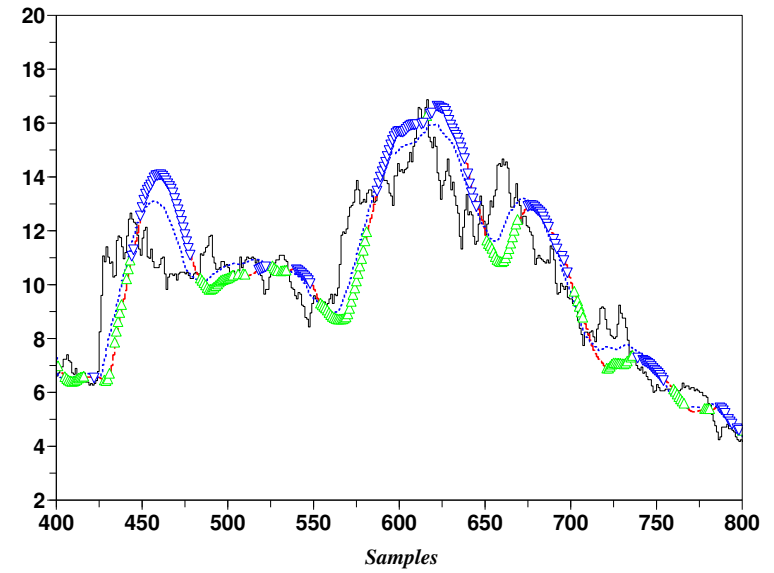

(a) Zoom of 6

Figure 7. Zoom of Figure 6 (5 days)

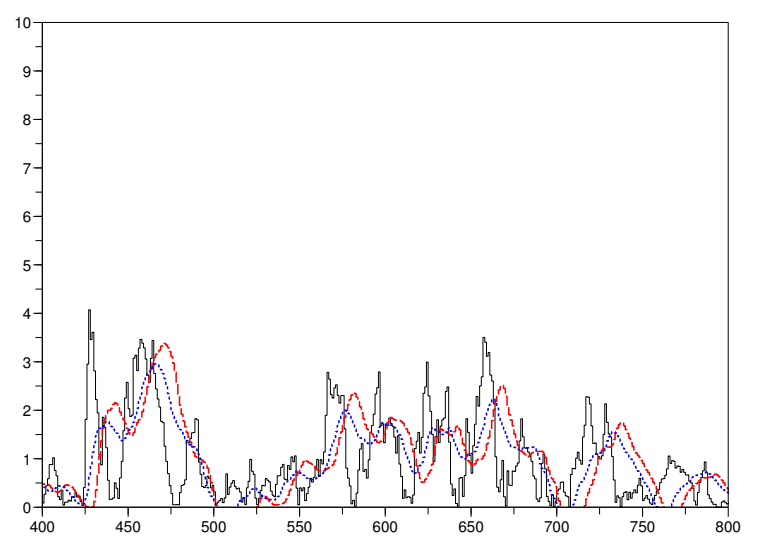

Figure 8. ABS-DIFF 5 (black,-), filtered signal (blue,.), 5 days forecast (red, - -) (5 days)

E.T. Jaynes. Probability Theory: The Logic of Science. Cambridge University Press, 2003.

P. Jorion. Value at Risk ( $2^{\text {nd }}$ ed.). Springer, 2001.

P.J. Kaufman. New Trading Systems and Methods ( $4^{\text {th }}$ ed.). Wiley, 2005.

C.D. Kirkpatrick, J.R. Dahlquist. Technical Analysis: The Complete Resource for Financial Market Technicians. FT Press, 2006.

A.W. Lo, J. Hasanhodzic. The Heretics of Finance: Conversations with the Leading Practitioners of Technical Analysis. Bloomberg, 2009.

C. Lobry, T. Sari. Nonstandard analysis and representation of reality. Int. J. Control, 81:517-534, 2008.

Y. Malevergne, D. Sornette. Extreme Financial Risks From Dependance to Risk Management. Springer, 2006.

B.B. Mandelbrot, R.L. Hudson. The (Mis)Behavior of Markets. Basic Books, 2004.

M. Mboup, C. Join, M. Fliess. Numerical differentiation with annihilators in noisy environment. Numer. Algorithm., 50:439-467, 2009.

C. Mouchot. Méthodologie économique. Hachette, 1996. 


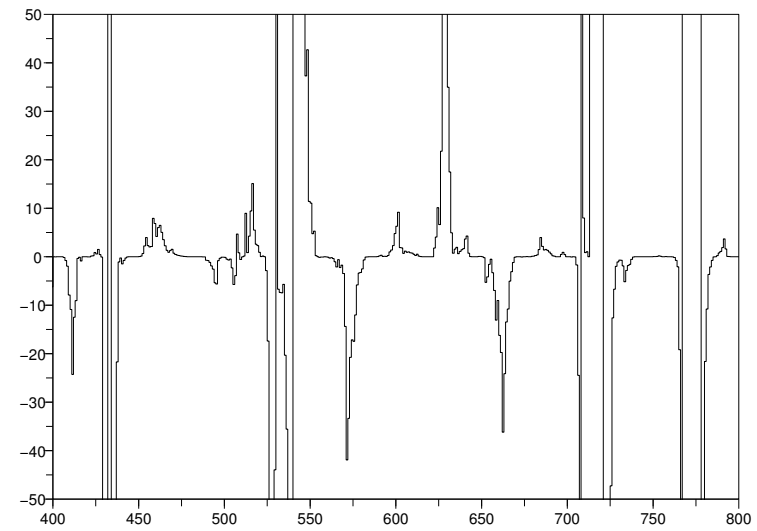

Figure 9. $\mathrm{SKEW}_{5}$ (5 days)

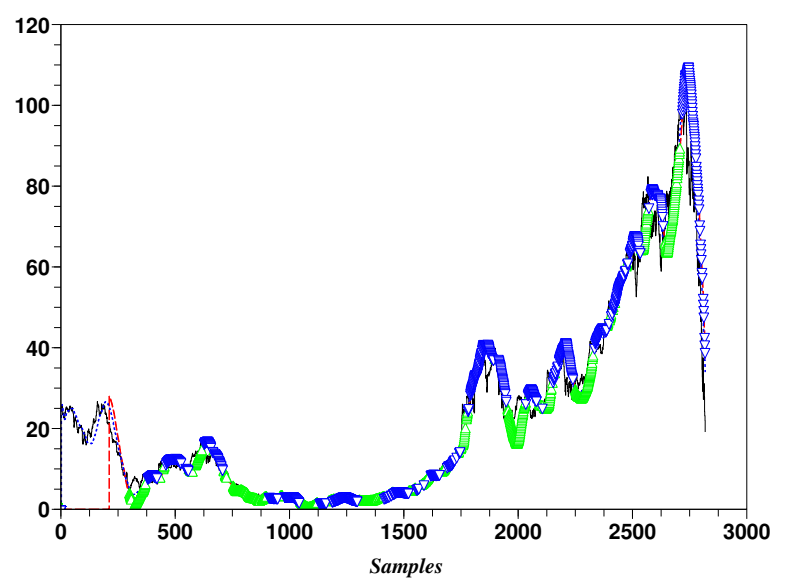

Figure 10. Prices (black,-), filtered signal (blue,.), 10 day forecast (red, - -), price's forecast higher than the predicted trend (green $\triangle$ ), price's forecast lower than the predicted trend (blue $\nabla)(10$ days)

J.J. Murphy. Technical Analysis of the Financial Markets ( $3^{\text {rd }}$ rev. ed.). New York Institute of Finance, 1999.

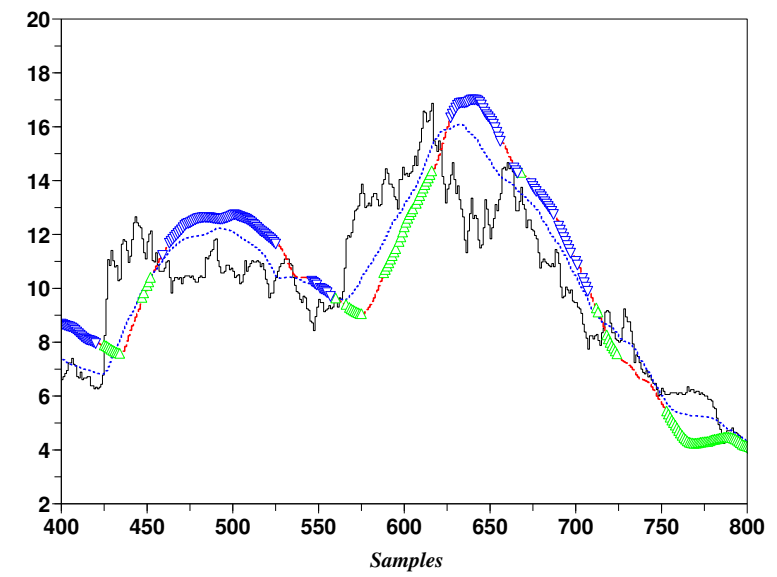

Figure 11. Zoom of Figure 10 (10 days)

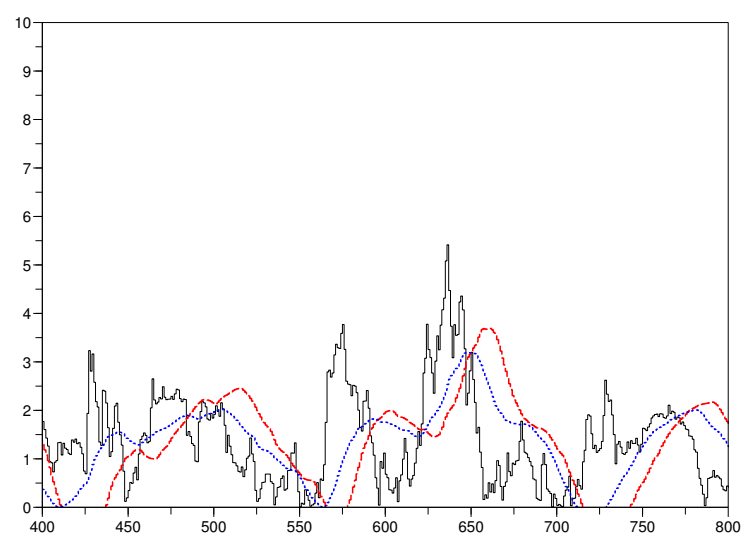

Figure 12. ABS-DIFF 10 (black,-), filtered signal (blue,.), 10 days forecast (red, - -) (10 days)

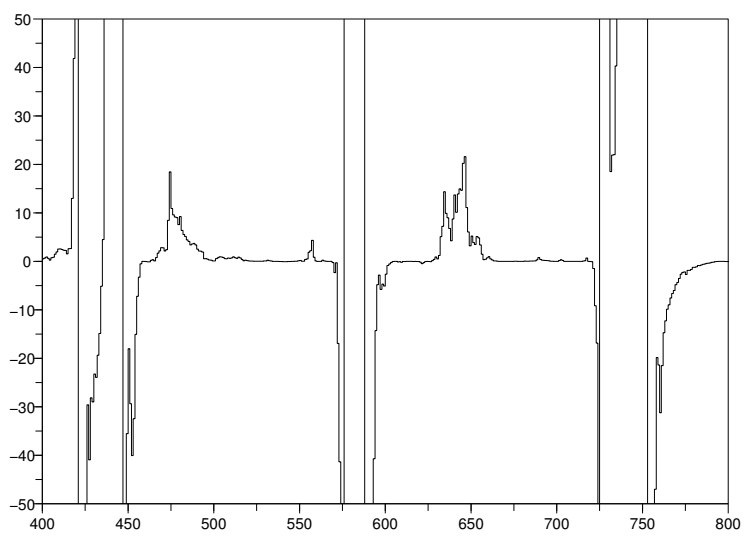

Figure 13. SKEW 10 (10 days) 


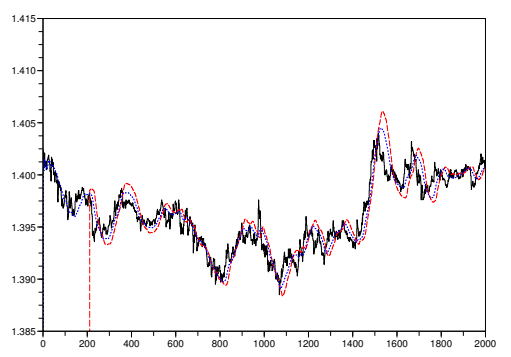

(a) Prices (black,-), filtered signal (blue,.), 10 days forecast (red, - -)

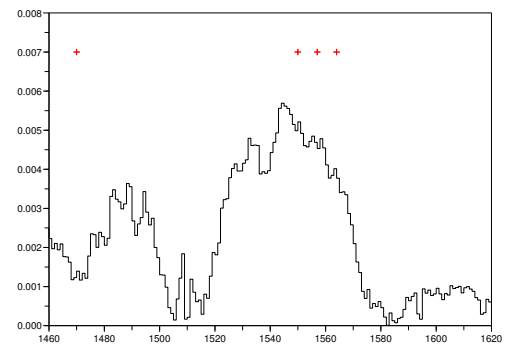

(d) Zoom of Figure 14-(b)

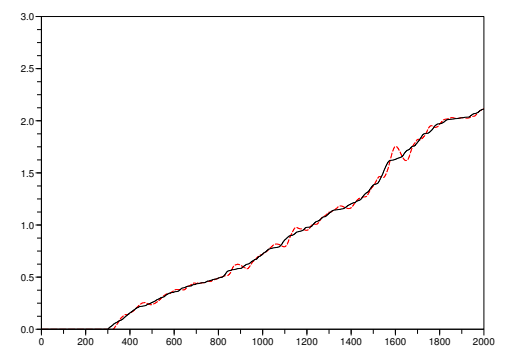

(b) $\int \mathrm{ABS}-\mathrm{DIFF}_{0}$ (black,-) and 5 days fore- (c) cast (red, - -)

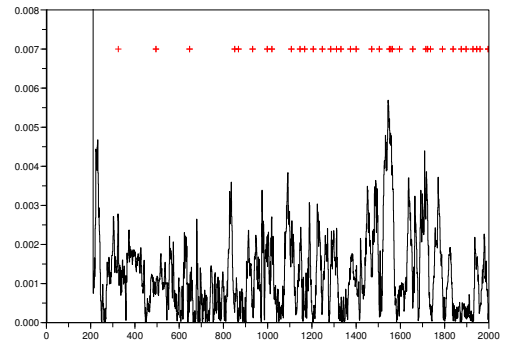

(e) $\int \mathrm{ABS}^{-\mathrm{DIFF}_{0}}$ (black,-), 10 days forecast (red, - -) and abrupt change location (red,+)

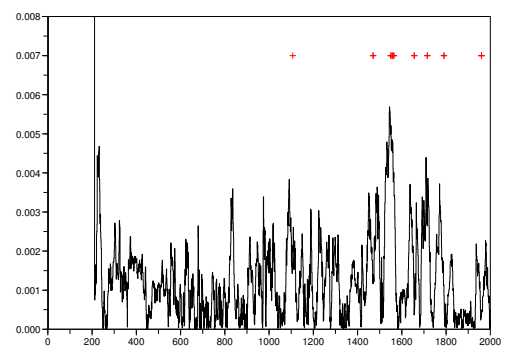

(c) $\int \mathrm{ABS}-\mathrm{DIFF}_{0}$ (black,-), 10 days forecast (red, - -) and abrupt change location (red, + )

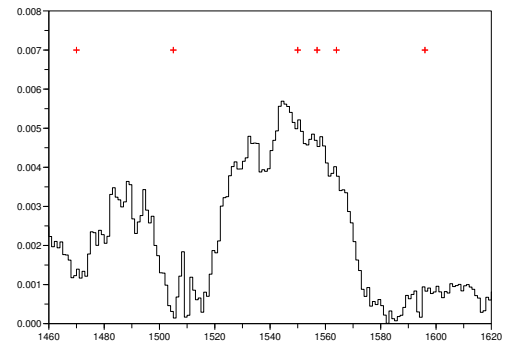

(f) Zoom of Figure 14-(e)

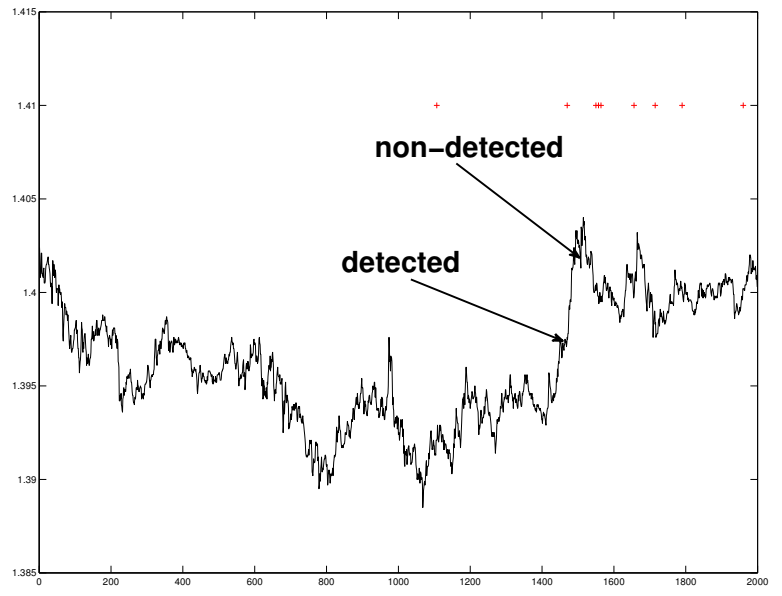

(g) Prices (black,-) and abrupt change location (red,+)

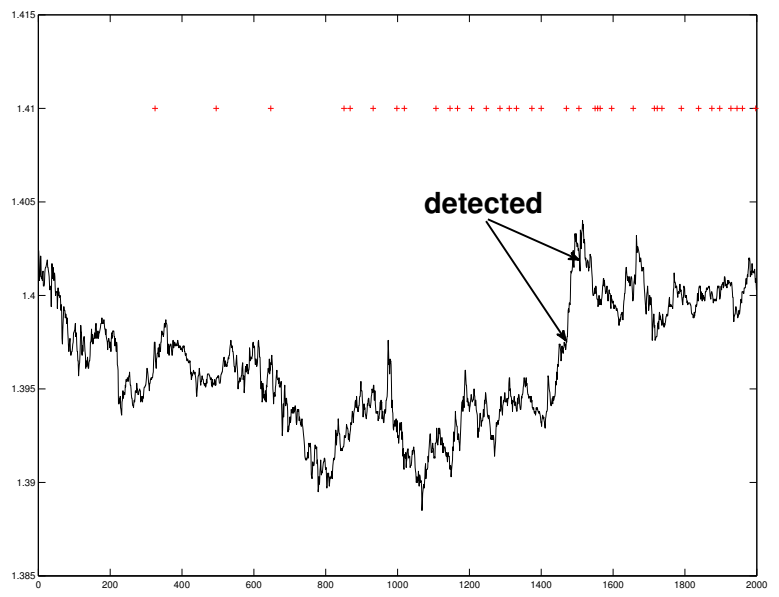

(h) Prices (black,-) and abrupt change location (red,+)

Figure 14. Daily exchange rate USD - EUR: 10 days forecasting 